\title{
Anti-Galectin-2 Antibody Treatment Reduces Atherosclerotic Plaque Size and Alters Macrophage Polarity
}

\author{
Jamie Kane 1,2,3,* Matthijs Jansen ${ }^{2,4, *}$ Sebastian Hendrix ${ }^{2}$ Laura A. Bosmans ${ }^{2}$ Linda Beckers ${ }^{2}$ \\ Claudia van Tiel $^{2}$ Marion Gijbels ${ }^{2,5}$ Noam Zelcer $^{2}$ Carlie J. de Vries ${ }^{2}$ Philipp von Hundelshausen ${ }^{6(0)}$ \\ Marc Vervloet $^{1}$ Ed Eringa ${ }^{3}$ Anton J. Horrevoets ${ }^{7}$ Niels van Royen ${ }^{8, *}$ Esther Lutgens ${ }^{2,6,9,10}$ \\ 1 Department of Nephrology, Amsterdam Cardiovascular Sciences, \\ Amsterdam University Medical Centre, Amsterdam, The Netherlands \\ 2 Department of Medical Biochemistry, Amsterdam Cardiovascular \\ Sciences, Amsterdam University Medical Centre, Amsterdam, The \\ Netherlands \\ 3 Department of Physiology, Amsterdam Cardiovascular Sciences, \\ Amsterdam University Medical Centre, Amsterdam, The Netherlands \\ ${ }^{4}$ Department of Cardiology, Amsterdam Cardiovascular Sciences, \\ Amsterdam University Medical Centre, Amsterdam, The Netherlands \\ ${ }^{5}$ Department of Pathology, Cardiovascular Research Institute \\ Maastricht (CARIM), GROW-School for Oncology and \\ Developmental Biology, Maastricht University, Maastricht, The \\ Netherlands \\ 6 Institute for Cardiovascular Prevention (IPEK), Ludwig- \\ Maximillian's University, Munich, Germany

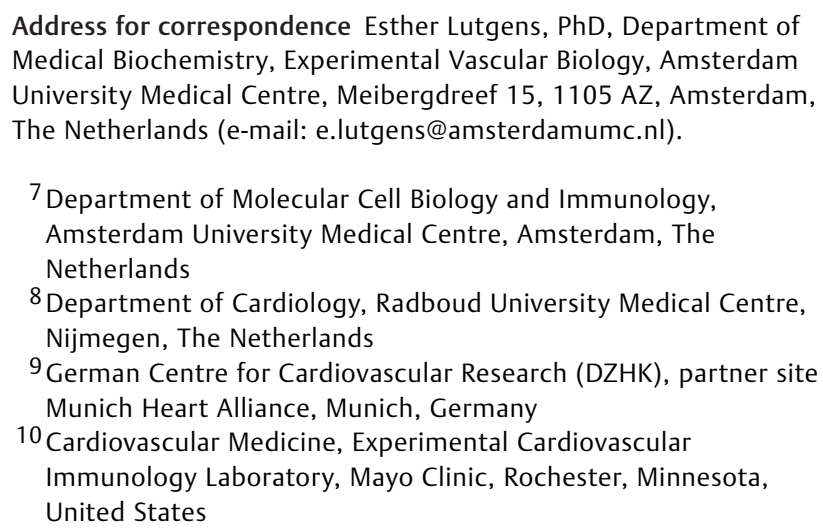

Address for correspondence Esther Lutgens, PhD, Department of Medical Biochemistry, Experimental Vascular Biology, Amsterdam University Medical Centre, Meibergdreef 15, 1105 AZ, Amsterdam, The Netherlands (e-mail: e.lutgens@amsterdamumc.nl).

${ }^{7}$ Department of Molecular Cell Biology and Immunology, Amsterdam University Medical Centre, Amsterdam, The Netherlands

${ }^{8}$ Department of Cardiology, Radboud University Medical Centre, Nijmegen, The Netherlands

${ }^{9}$ German Centre for Cardiovascular Research (DZHK), partner site Munich Heart Alliance, Munich, Germany

${ }^{10}$ Cardiovascular Medicine, Experimental Cardiovascular Immunology Laboratory, Mayo Clinic, Rochester, Minnesota, United States

Thromb Haemost 2022;122:1047-1057.
Abstract
Keywords
- nanobodies
- anti-inflammatory agents
- atherosclerosis
- galectin-2
- macrophages

Background Galectins have numerous cellular functions in immunity and inflammation. Short-term galectin-2 (Gal-2) blockade in ischemia-induced arteriogenesis shifts macrophages to an anti-inflammatory phenotype and improves perfusion. Gal-2 may also affect other macrophage-related cardiovascular diseases.

Objectives This study aims to elucidate the effects of Gal-2 inhibition in atherosclerosis.

Methods ApoE ${ }^{-1-}$ mice were given a high-cholesterol diet (HCD) for 12 weeks. After 6 weeks of $\mathrm{HCD}$, intermediate atherosclerotic plaques were present. To study the effects of anti-Gal-2 nanobody treatment on the progression of existing atherosclerosis, treatment with two llama-derived anti-Gal-2 nanobodies (clones $2 \mathrm{H} 8$ and $2 \mathrm{C} 10$ ), or vehicle was given for the remaining 6 weeks.

Results Gal-2 inhibition reduced the progression of existing atherosclerosis. Atherosclerotic plaque area in the aortic root was decreased, especially so in mice treated with 2C10 nanobodies. This clone showed reduced atherosclerosis severity as reflected by a decrease in fibrous cap atheromas in addition to decreases in plaque size.

\footnotetext{
* Shared authorship.
}

received

November 5, 2020 accepted after revision September 26, 2021 published online December 1, 2021
DOI https://doi.org/ 10.1055/a-1711-1055. ISSN 0340-6245. (c) 2021. The Author(s).

This is an open access article published by Thieme under the terms of the Creative Commons Attribution-NonDerivative-NonCommercial-License, permitting copying and reproduction so long as the original work is given appropriate credit. Contents may not be used for commercial purposes, or adapted, remixed, transformed or built upon. (https://creativecommons.org/ licenses/by-nc-nd/4.0/) Georg Thieme Verlag KG, Rüdigerstraße 14, 70469 Stuttgart, Germany 
The number of plaque resident macrophages was unchanged; however, there was a significant increase in the fraction of $\mathrm{CD}^{206} 6^{+}$macrophages. $2 \mathrm{C} 10$ treatment also increased plaque $\alpha$-smooth muscle content, and Gal- 2 may have a role in modulating the inflammatory status of smooth muscle cells. Remarkably, both treatments reduced serum cholesterol concentrations including reductions in very low-density lipoprotein, low-density lipoprotein, and high-density lipoprotein while triglyceride concentrations were unchanged.

Conclusion Prolonged and frequent treatment with anti-Gal-2 nanobodies reduced plaque size, slowed plaque progression, and modified the phenotype of plaque macrophages toward an anti-inflammatory profile. These results hold promise for future macrophage modulating therapeutic interventions that promote arteriogenesis and reduce atherosclerosis.

\section{Introduction}

Atherosclerosis is a chronic inflammatory disease which progresses slowly with a build-up of lipid-laden plaques in medium- and large-sized arteries. ${ }^{1}$

Galectins are a family of $\beta$-galactoside-specific lectins, which signal by cross-linking glycoproteins on cell membranes. Galectin subtypes have distinct roles in the immune system, inflammation, and wound healing. ${ }^{2}$ Each galectin has unique carbohydrate recognition domains ${ }^{3}$ and preferentially binds to specific glycoproteins or glycoconjugates to mediate cellular functions. ${ }^{4,5}$

Galectin-2 (Gal-2) exists as a dimer and is expressed in the stomach and small intestine, specifically in the upper region of the intestinal crypts, bottom of the villi, and in goblet cells. ${ }^{6}$ Recently, Gal-2 has attracted interest in immunology and various disease contexts. Gal-2 suppresses contact allergies by inducing apoptosis in activated $\mathrm{CD}^{+} 4$ and $\mathrm{CD}^{+}{ }^{+} \mathrm{T}$ cells, ${ }^{7}$ and ameliorates acute and chronic inflammatory bowel disease (IBD) in mice. ${ }^{8}$ Relatedly, Gal-2 can inhibit the secretion of Th1 (interferon [IFN]gamma and interleukin [IL]-2) and Th17 (IL-17) type cytokines and potentiate secretion of Th2 (ILs 4, 5, 10, and 13) type cytokines. ${ }^{5}$

Arteriogenesis is the protective response whereby preexisting collateral arteries remodel and increase their diameter to compensate for arterial stenosis. ${ }^{9,10}$ Gal-2 messenger ribonucleic acid (mRNA) expression is increased in the monocytes and macrophages of patients with a low arteriogenic response, and arteriogenesis is impaired by Gal-2 in a murine hind limb model. ${ }^{11}$ Gal-2 reduces the number of perivascular macrophages around vessels undergoing arteriogenesis. ${ }^{11}$ Furthermore, the rs7291467 single-nucleotide polymorphism associates to the highest Gal-2 macrophage mRNA expression and impaired arteriogenesis, which demonstrates genetic influences. ${ }^{11}$

Yildirim et al characterized the mechanism of action of Gal-2. ${ }^{12}$ Their study showed that Gal-2 binds CD14 on the surface of human monocytes and monocyte-derived macrophages from healthy donors, and its downstream activity is induced via Toll-like receptor 4 . Gal-2 polarizes macrophages to the M1 proinflammatory subtype, resulting in increased expression of tumor necrosis factor- $\alpha$ (TNF- $\alpha$ ), IL-6, and IFN$\beta$. Gal-2 also suppresses anti-inflammatory M2 macrophage differentiation, which is reflected by a reduced expression of the key anti-inflammatory cytokine transforming growth factor- $\beta-1 .^{12}$

Recently, Hollander et al $^{13}$ developed the first anti-Gal-2 nanobodies. Their approach was to immunize llamas with human and mouse recombinant Gal-2. The llamas were then used to isolate variable domain of heavy chain ( $\mathrm{VHH}$ ) genes raised against Gal-2 and using a bacterial phage "panning" method a selection of specific clones was selected, giving an initial candidate pool of 184 clones. Following Gal-2 binding and monocyte binding assays this pool was narrowed down to five. Four of these had unique sequences, and after assessments of binding specificity to Gal-2, the 2H8 and 2C10 clones were finally selected as the most suitable for in vivo experiments.

The effects of their two selected clones on arteriogenesis and macrophage polarity were tested. A "hind limb ischemia" model was utilized where the right femoral artery of mice was ligated and the response over the following 14 days was assessed. 2H8 and 2C10 nanobody treatment significantly promoted arteriogenesis resulting in improved collateral artery growth and increased perfusion restoration. Both nanobodies strongly inhibited Gal-2 macrophage binding and shifted the ratio of M1:M2 macrophages toward a preponderance of anti-inflammatory M2 macrophages. As a result, Gal-2-induced genes were reduced, including TNF- $\alpha$, IL-6, and IFN- $\beta$.

In this study, we utilize the $2 \mathrm{H} 8$ and $2 \mathrm{C} 10$ nanobody clones to study the role of Gal-2 in atherosclerosis. Genetic association and histopathological studies have demonstrated that Gal-2 is associated with the presence and severity of atherosclerotic disease. ${ }^{14}$ To evaluate Gal-2 as a potential target for anti-atherosclerotic therapies, we investigate the effects of anti-Gal-2 nanobodies on the development and progression of atherosclerosis in $A p o E$ -l- mice. 


\section{Methods}

\section{Production of Llama Antibodies}

Recombinant human and murine Gal-2 was produced ${ }^{12}$ and used to immunize llamas. 2H8 and 2C10 llama-derived antiGal-2 nanobodies were produced as previously described by Hollander et al. ${ }^{13}$

The $2 \mathrm{H} 8$ and $2 \mathrm{C} 10$ clones were selected for this study, with each nanobody being specific to a different epitope of Gal-2.

\section{Animal Experiments}

Apo $E^{-/-}$mice were purchased from Janvier and were housed and bred according to institutional guidelines. Animal experiments were approved by the institutional Animal Experimental Ethics committee and the National Central Committee on Animal Experimentation (DBC242AJ), in conformation with directive 2010/63/EU of the European Parliament. $A p o E^{-/-}$mice were used to test the effects of the $2 \mathrm{H} 8$ and 2C10 clones (previously developed by Hollander et $\mathrm{al}^{13}$ ) of the anti-Gal-2 nanobody on atherosclerosis. Mice received a high cholesterol (HC; $0.15 \%$, Altromin, Lage, Germany) diet from 10 weeks of age which then continued for 12 weeks.

Following 6 weeks of HC diet, mice were treated with nanobody $2 \mathrm{H} 8,2 \mathrm{C} 10$, or the vehicle control (saline) four times a week for 6 weeks via intraperitoneal injection. The injection frequency was determined based on the high clearance rate of nanobodies. ${ }^{13,15}$ The charging dose was $0.51 \mathrm{mg}$ of $2 \mathrm{H} 8$ or 2C10 nanobody diluted in saline solution for every mouse. Maintenance dose was $0.015 \mathrm{mg}$ for both antibodies. Control mice were given isovolumetric intraperitoneal injections of sterile saline. A vehicle control was utilized in place of an irrelevant nanobody to equalize the effect of injections between groups as the effect of another nanobody would be unclear.

At the conclusion of the experiment mice were euthanized by $\mathrm{CO}_{2}$ gassing. Subsequently, blood was obtained via cardiac puncture and the arterial tree was perfused with sterile phosphate-buffered saline and fixed in $1 \%$ paraformaldehyde (PFA). All organs were harvested, fixed overnight with 4\% PFA, dehydrated, and embedded in paraffin.

\section{Histology}

Twenty-five serial sections of the heart (beginning $4 \mu \mathrm{m}$ apart), with between four and six levels (and each level therefore $100 \mu \mathrm{m}$ apart) were cut. Sections began when the aortic valve became visible and continued until it was no longer visible. Sections 8 and 16 from each mouse were analyzed for plaque area. Each section gives a view throughout the whole aortic root. Lymph nodes, spleens, intestines, and livers were cut in $4 \mu \mathrm{m}$ sections. Chemical stainings were as follows-all organs were stained with hematoxylin and eosin, and selected sections of the aortic root were stained with Picro-Sirius red to visualize plaque collagen content.

Immunohistochemical stainings were as follows-tissue sections were rehydrated in xylene and $100 \%$ ethanol. Slides were then pretreated with $0.3 \% \mathrm{H}_{2} \mathrm{O}_{2}$ in absolute methanol for 30 minutes, followed by a minute in $100,96,70$, and $50 \%$ ethanol. Next, slides were treated with $1 \times$ sodium citrate antigen retrieval buffer at approximately $95^{\circ} \mathrm{C}$ for 10 minutes. Sections were then incubated with their primary antibody, these were anti- $\alpha$-smooth muscle actin $(\alpha \mathrm{SMA})^{\mathrm{FITC}}$ (clone 1A4, 1:3000; Sigma-Aldrich, United States), anti-Mac3 (M3/84, 1:100; BD Pharmingen, United States), anti-CD206 (polyclonal, 1:1000; Abcam, United Kingdom), anti-ABCA-1 (polyclonal, 1:200; Novus Biologicals, United States), and anti-ABCG-1 (polyclonal, 1:1000; Novus Biologicals).

Primary antibody binding was detected with the appropriate secondary antibody, these were anti-FITC ${ }^{\text {Biotin }}$ (1F8-1E4, 1:600, Jackson Immunoresearch, United Kingdom) for $\alpha$ SMA, anti-rat immunoglobulin (Ig) G (polyclonal, 1:300, Vector Labs, United States) for Mac3, and anti-rabbit Ig/Biotin (polyclonal, 1:300, Dako, United States) for CD206, ABCA-1, and ABCG-1. Next slides were incubated with the $A B C$ kit according to the manufacturer's instructions (Vector Labs), and finally immunoreactivity was visualized using 3,3'diaminobenzidine (Dako) for all except Mac3 where ImmPACT Vector Red Substrate (Vector Labs) was used. Lastly, slides were counterstained with hematoxylin and mounted with Entellan (Merck, Darmstadt, Germany). All steps were at room temperature and diluted in Tris-buffered Tween (Trisbuffer saline [TBS] - $1 \%$ bovine serum albumin - $0.1 \%$ Tween) where applicable.

\section{Microscopy and Plaque Analysis}

Images were acquired using a Leica DM5500B fluorescence light microscope (Leica Microsystems, Wetzlar, Germany) equipped with a DCF295 camera with $5 \times$ or $10 \times$ lens as indicated. Plaque area and positively stained areas were assessed using ImageJ (National Institutes of Health, United States) and Adobe Photoshop (Adobe Inc, United States), respectively. Areas positive for the protein of interest were analyzed by applying color threshold measurements based upon a positive control.

The aortic root plaques were categorized using the Virmani classification. ${ }^{16}$ Briefly, each plaque was assigned to one of three categories. First, the most advanced is a fibrous cap atheroma (FCA)-plaques containing an established necrotic core with an overlaying fibrous cap. Second, and less advanced than FCAs are pathological intimal thickenings (PITs)-plaques with areas of extracellular lipid accumulation but no necrotic core. Lastly, and least advanced are intimal thickenings-plaques with an accumulation of smooth muscle cells (SMCs) in the intima. Total number of plaques of each category is shown for each group. To remove bias plaques were blinded and assessed by a single operator.

\section{Cholesterol and Triglyceride Measurement}

Cholesterol and triglyceride concentration in mice serum was measured using the CHOD PAP method and GPO PAP method kits (BioLabo, France), respectively, according to the manufacturer's instructions. Microplates were read at $485 \mathrm{~nm}$ using an Epoch microplate spectrophotometer (BioTek, United States). Results were analyzed by Gen5 v3.05 software (BioTek). 
Concentrations of different cholesterol fractions, highdensity lipoprotein (HDL), low-density lipoprotein (LDL), and very low-density lipoprotein (VLDL) were determined using fast protein liquid chromatography as described previously. ${ }^{17}$ In brief, the system consisted of a PU-980 ternary pump with an LG-980-02 linear degasser and a UV-975 UV/VIS detector (Jasco, Japan). First, $30 \mu \mathrm{L}$ of plasma was injected and lipoproteins were separated using a Superose 6 Increase 10/300 column (GE Healthcare, the Netherlands). TBS ( $\mathrm{pH}$ 7.4) was used as eluent with a flow rate of 0.31 $\mathrm{mL} / \mathrm{min}$. Next, an auxiliary pump (PU-2080i Plus; Jasco) was used for in-line cholesterol PAP substrate reagent (Sopachem, the Netherlands), addition at a flow rate of $0.1 \mathrm{~mL} / \mathrm{min}$. Following lipoprotein detection, lipid plasma standards (SKZL, the Netherlands) were used to generate calibration curves. The sample and calibration data were combined and quantitative analysis of the fractions was performed to give final concentrations using Chrom-Nav chromatographic software, version 2.0 (Jasco).

\section{In Vitro Hepatocyte Experiments}

HepG2 cells (American Type Culture Collection, Manassas, Virginia, United States) were cultured in Dulbecco's modified Eagle's medium (DMEM; Gibco, United States) supplemented with $10 \%$ fetal calf serum (FCS; Gibco), 100 units/mL penicillin, and $100 \mu \mathrm{g} / \mathrm{mL}$ streptomycin (Gibco) at $37^{\circ} \mathrm{C}$ and $5 \% \mathrm{CO}_{2}$. For sterol depletion, cells were cultivated in DMEM supplemented with $10 \%$ lipoprotein-deficient serum, $100 \mu \mathrm{M}$ mevalonic acid, and $2.5 \mu \mathrm{g} / \mathrm{mL}$ simvastatin for 24 hours.

RNA was isolated from HepG2 cells using a Direct-zol RNA MiniPrep kit (Zymo Research, United States) according to the manufacturer's instructions. Note that $1 \mu \mathrm{g}$ of total RNA was reverse transcribed into complementary deoxyribonucleic acid (cDNA) using the iScript cDNA synthesis kit (BioRad, United States). Real-time quantitative polymerase chain reaction (PCR) was performed on a LightCycler 480 II system (Roche, Switzerland) using SensiFAST SYBR (Bioline, United Kingdom). Gene expression levels were normalized to the averaged expression of 36B4 and glyceraldehyde 3-phosphate dehydrogenase and are presented as fold change values which are calculated using the $\mathrm{ddCt}$ method.

\section{In Vitro Smooth Muscle Cell Experiments}

Human SMCs were explanted from umbilical cord arteries. Cells were cultured in DMEM (Gibco) supplemented with 10\% FCS (Gibco), 100 units/mL penicillin, and $100 \mu \mathrm{g} / \mathrm{mL}$ streptomycin (Gibco) at $37^{\circ} \mathrm{C}$ and $5 \% \mathrm{CO}_{2}$.

SMC quiescence was induced by incubation for 24 hours in FCS-free medium. Cells were then stimulated with $10 \%$ FCS or indicated concentrations of Gal-2 for 4 or 24 hours. DNA synthesis was monitored by 5-bromo-2-deoxyuridine (BrdU) incorporation according to the manufacturer's protocol (Roche, Switzerland).

RNA was isolated from the SMCs using the RNeasy Mini Kit II (Qiagen, Germany). RNA was reverse transcribed with a High-Capacity cDNA Reverse Transcription Kit (Thermo Fisher, United States). Quantitative PCR was performed with a
Sybr Green Master mix (Life Technologies, United States) on a ViiA7 real-time PCR system (Life Technologies). Primers were purchased from Sigma-Aldrich and sequences are available upon request.

SMC and hepatocyte experiments used recombinant human Gal-2 protein (Novus Biologicals).

\section{Statistical Analysis}

Results from the analyses of histological stainings were compared using a one-way analysis of variance with a Dunnett's multiple comparisons test. Plaque classifications (i.e., categorical data) were compared using 2 two-sided Fisher's exact tests, with a Bonferroni correction (and therefore $p<0.025$ was considered significant). Analysis was performed using GraphPad Prism v9.1.0 (GraphPad Software Inc., United States).

\section{Results}

Treatment with $2 \mathrm{H8}$ and $2 \mathrm{C10}$ Antibodies Reduces Atherosclerosis and Induces a Favorable Atherosclerotic Plaque Phenotype

The progression of existing atherosclerotic plaques in $\mathrm{ApoE}^{-/}$ mice was delayed following treatment with anti-Gal-2 nanobodies. Both 2H8 and 2C10 nanobody treatments significantly decreased atherosclerotic plaque area in the aortic roots (-Fig. 1A-E).

While both $2 \mathrm{H} 8$ and $2 \mathrm{C} 10$ nanobodies are able to ameliorate atherosclerosis, the effects observed with 2C10 treatment were stronger. In addition to the effects on plaque size, ApoE ${ }^{-/-}$mice treated with 2C10 nanobody also prevented the transition of plaques from PITs to FCAs. Following 12 weeks of high-cholesterol diet (HCD), 2C10 was the only treatment able to significantly increase the number of PITs, signifying a less advanced atherosclerotic burden than control (-Fig. 1B).

Representative images ( - Fig. 1C-E) were selected to show aortic roots with plaque areas closest to the mean average of that group and their average classification. Additionally, treatment had no effect on mouse body weight. Control mice were $25.4 \mathrm{~g} \pm 3.55,2 \mathrm{H} 8$ treatment mice were $24.9 \mathrm{~g} \pm 2.49$, and $2 \mathrm{C} 10$ treatment mice were $25.2 \mathrm{~g} \pm 2.48$ $(p=0.872)$.

In summary, these data show mice treated with either Gal-2 antibody have reduced plaque size versus control, and exhibited a less advanced plaque phenotype when treated with $2 \mathrm{C} 10$.

\section{Anti-Galectin-2 Nanobody Treatments Modify the Composition of the Plaque}

As Gal-2 has been reported to play a key role in the inflammatory state of macrophages, ${ }^{12}$ we analyzed the effects of anti-Gal-2 treatment on atherosclerotic plaque macrophages. Total plaque macrophage content and the absolute number of Mac3 positive plaque macrophages were unchanged in both treatment groups (- Fig. 2A-D). Additional phenotyping of these macrophages revealed that $2 \mathrm{C} 10$ treatment increased the percentage of CD206+ macrophages in 

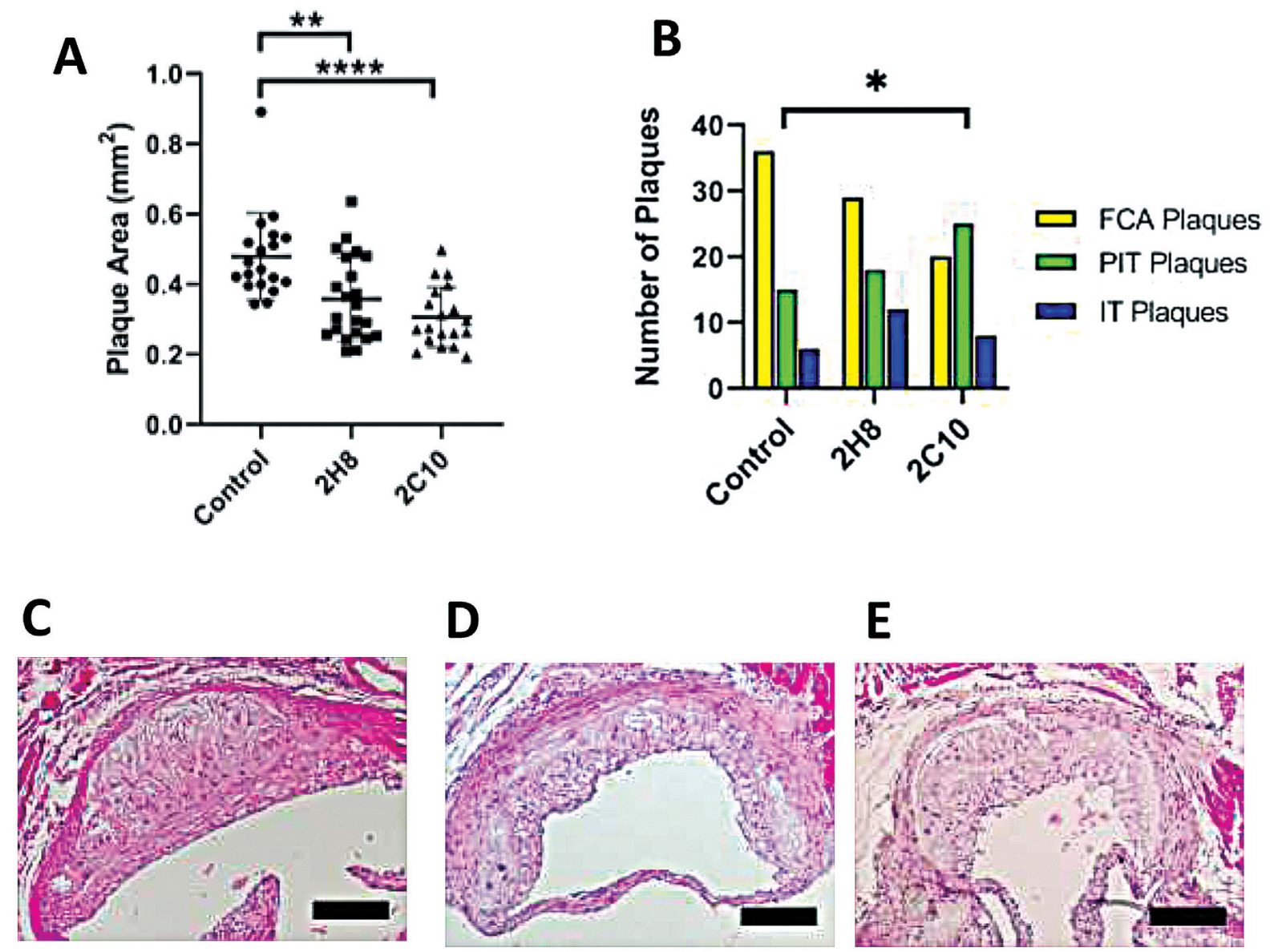

Fig. 1 Treatment with two anti-galectin-2 (Gal-2) antibodies, $2 \mathrm{H} 8$ and $2 \mathrm{C} 10$, reduces plaque area and changes the plaque phenotype. (A) Plaque area in the aortic roots of $A p o E^{-/-}$mice under control, $2 \mathrm{H} 8$, or $2 \mathrm{C} 10$ antibody treatments. Mean value with standard deviation, $n=19 .{ }^{*} p \leq 0.05$. ${ }^{* *} p \leq 0.01 .{ }^{* * *} p \leq 0.001 .{ }^{* * * *} p \leq 0.0001$. (B) Qualitative categorization of plaque phenotype following hematoxylin and eosin staining. FCA, fibrous cap atheroma; PIT, pathological intimal thickening; IT, intimal thickening. Fisher's exact test, $p=0.0129$. Representative images of aortic roots stained with hematoxylin and eosin under control (C), $2 \mathrm{H} 8$ (D), and 2C10 (E) conditions are shown. Scale bar is $150 \mu \mathrm{m}$.

the plaque, suggesting that $2 \mathrm{C} 10$ treatment polarizes macrophages to a more anti-inflammatory phenotype (-Fig. 2EH). Interestingly, $2 \mathrm{C} 10$ treatment was also able to change the expression of $\mathrm{ABC}$ transporters on plaque macrophages, showing an increase in ABCA- 1 and a concurrent decrease in ABCG-1 (-Fig. 2I-P). Anti-Gal-2 treatments had no effect on plaque necrosis, collagen content, or fibrous cap thickness (-Supplementary Fig. S1A-S1C, available in the online version).

We also assessed lymphoid organs to investigate any potential effects of nanobody treatment on wider systemic immune reactivity. Spleens of anti-Gal-2 treated mice did not show features of white pulp atrophy, extra medullary hematopoiesis, or marginal zone atrophy (-Supplementary Fig. S1E-S1G, available in the online version). $\mathrm{T}$ cell and B cell zones in the spleen (as assessed by CD3 and B220 stainings) were well developed (-Supplementary Fig. S2A-S2F, available in the online version). However, $2 \mathrm{C} 10$ treated mice did show a minor increase in the number of splenic macrophages as shown in - Supplementary Fig. S1H-S1J (available in the online version).

\section{Anti-Galectin-2 Nanobody Treatment Stabilizes Plaques}

In addition to these increases in CD206+ macrophages in the plaque, 2C10 treatment resulted in an increase in $\alpha \mathrm{SMA}+$ vascular SMCs (VSMCs) (-Fig. 3A-D). This suggests the less advanced plaque phenotype we observed also exhibits slight increases in plaque stability. Therefore, we investigated if Gal-2 had a direct effect on VSMC proliferation and/or inflammation by stimulating human VSMCs with recombinant Gal-2 protein and assessing their response. Gal-2 does not affect VSMC proliferation as shown by a BrdU incorporation assay (-Fig. 3E). However, IL-1 $\beta$ and chemokine (C-C motif) ligand 2 (CCL2) expression is increased while TNF- $\alpha$ expression is unchanged after stimulation with $10 \mu \mathrm{g} / \mathrm{mL}$ of Gal-2 (-Fig. 3F-H).

Taken together, these data show that there is more $\alpha \mathrm{SMA}$ in the aortic root plaques (localized to the fibrous cap) of mice treated with the $2 \mathrm{C} 10$ anti-Gal-2 nanobody. Additionally, Gal-2 has no effect on SMC proliferation and has a mild effect on the expression of inflammatory genes following VSMC stimulation. 
A

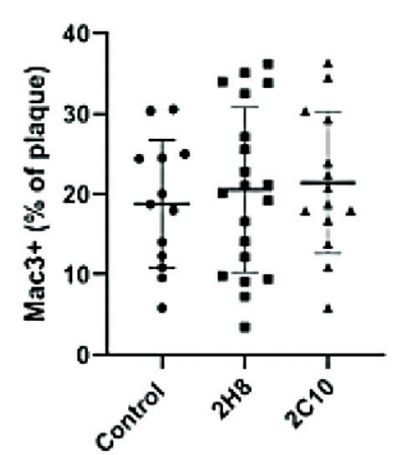

$\mathbf{E}$
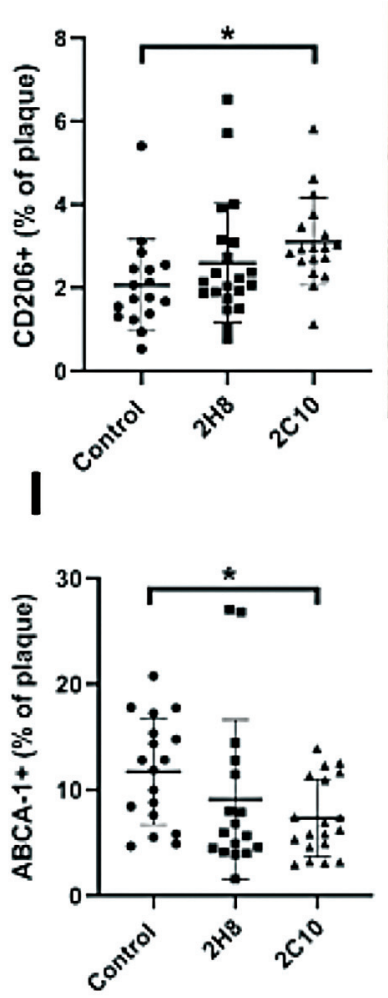

M

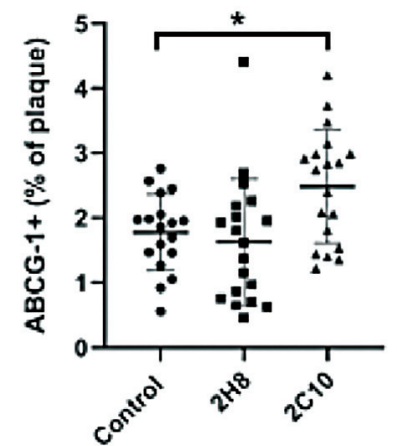

B

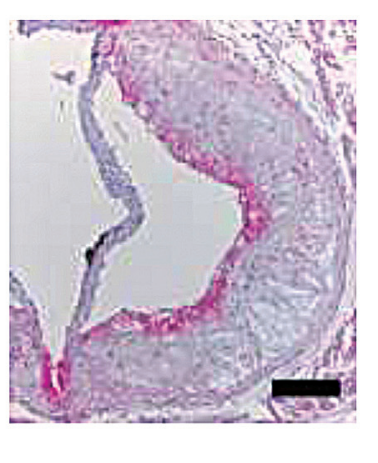

F
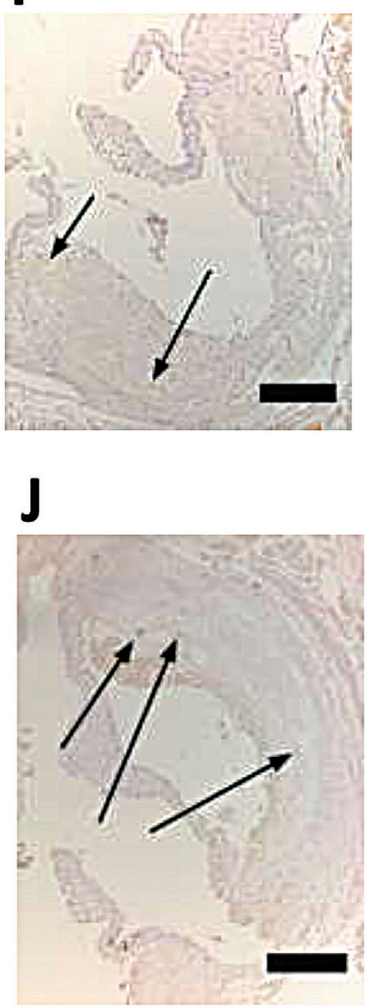

N

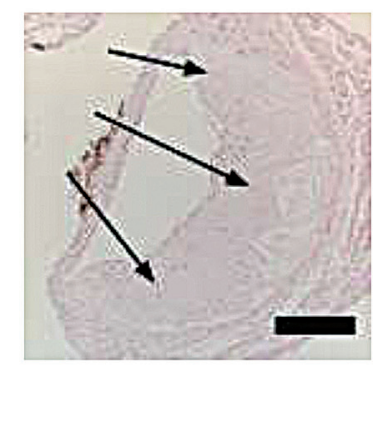

C

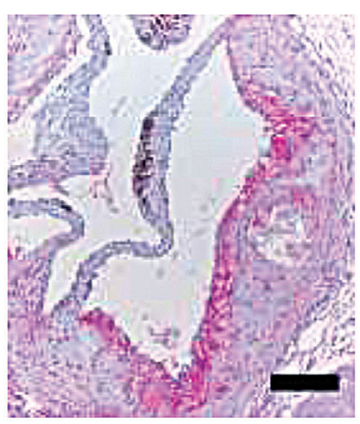

G

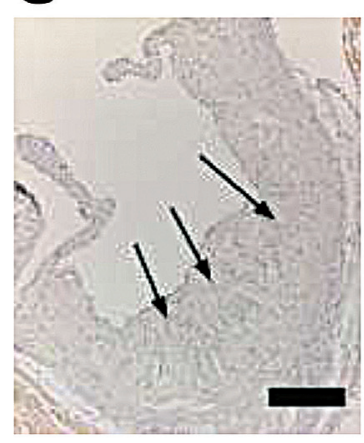

K

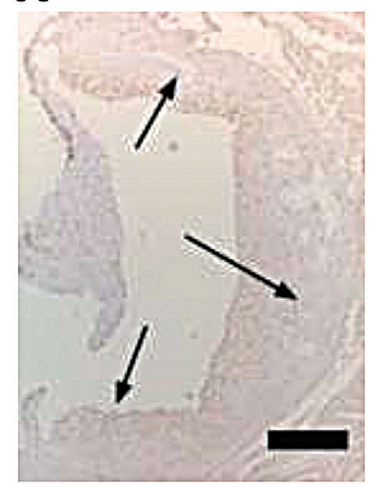

0

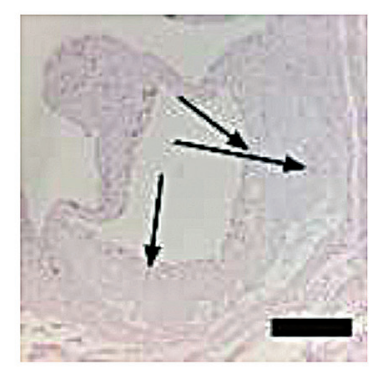

D

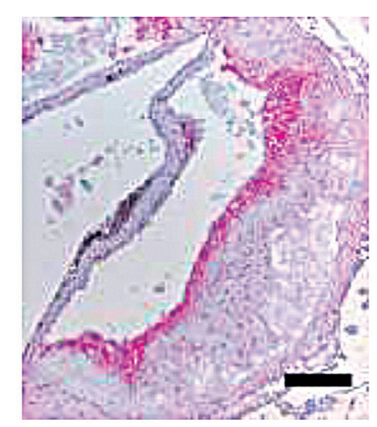

H

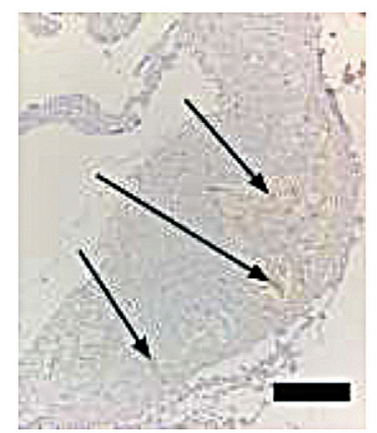

L

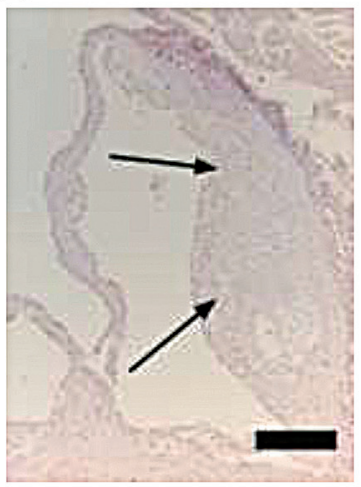

P

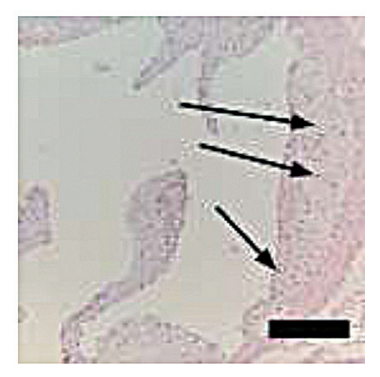

Fig. 2 An increased ratio of macrophages in the plaque is anti-inflammatory with modified cholesterol efflux regulatory protein levels. Percentage of the plaques staining positive for the macrophage marker, Mac3 (A), the anti-inflammatory macrophage marker CD206 (E), ABCA-1 (I), and ABCG-1 (M) are shown. Representative pictures of each stain from control, 2H8, and 2C10 treatment groups, respectively, are shown (B$\mathrm{D}, \mathrm{F}-\mathrm{H}, \mathrm{N}-\mathrm{P}) .{ }^{*} \mathrm{P} \leq 0.05 .{ }^{* *} \mathrm{p} \leq 0.01 .{ }^{* * *} p \leq 0.001 .{ }^{* * * *} p \leq 0.0001$. Scale bars represent $150 \mu \mathrm{m}$. 

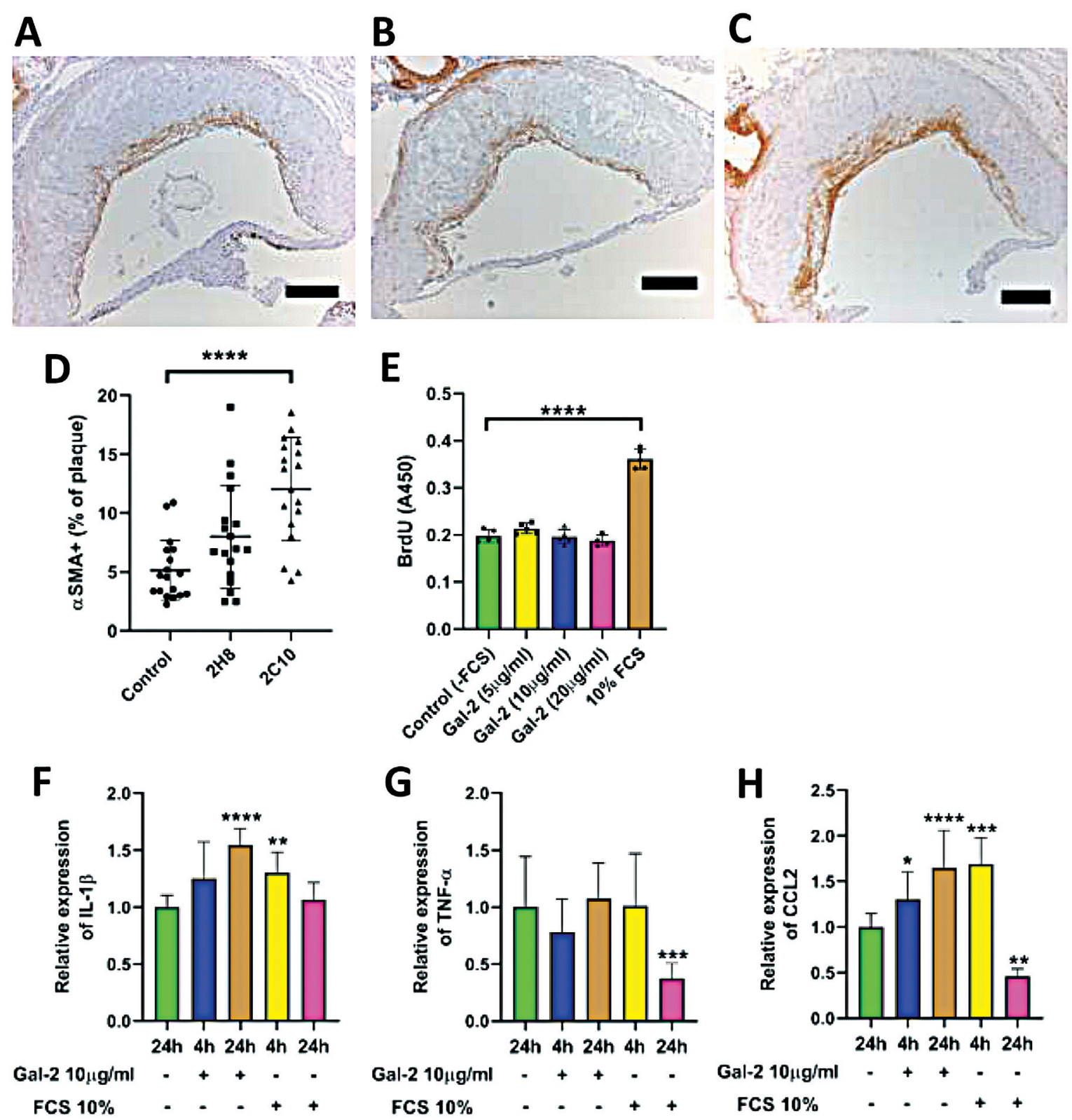

Fig. 3 There is more smooth muscle actin present in the plaques of anti-galectin-2 (Gal-2) treated mice, yet galectin-2 has no effect on smooth muscle cell proliferation and a mild inflammatory effect. Representative images of control (A), $2 \mathrm{H} 8$ (B), and $2 \mathrm{C} 10$ (C) conditions. Scale bars represent $150 \mu \mathrm{m}$. Percentage of plaque area positive for $\alpha$-smooth muscle actin ( $\alpha \mathrm{SMA}$ ) (D) under control, $2 \mathrm{H} 8$, and $2 \mathrm{C} 10$ conditions. Mean values with standard deviation. $n=19$. 5-bromo-2-deoxyuridine (BrdU) incorporation assay ( $E$ ) to measure vascular smooth muscle cell (VSMC) proliferation under different concentrations of galectin-2. $n=5$. Relative quantitative polymerase chain reaction (qPCR) gene expression vs. control of three markers of inflammation, interleukin (IL)-1 $\beta$ (F), tumor necrosis factor- $\alpha$ (TNF- $\alpha)(G)$, and CCL2 (H) stimulated with galectin-2 for 4 and 24 hours. ${ }^{*} p \leq 0.05 .{ }^{* *} p \leq 0.01 .{ }^{* * *} p \leq 0.001 .{ }^{* * * *} p \leq 0.0001$. Standard deviation shown, $n=3$.

\section{Anti-Gal-2 Treatment Reduces Plasma Cholesterol Levels}

Analysis of total cholesterol and triglyceride levels revealed that anti-Gal-2 nanobody treatment reduced serum cholesterol levels while triglyceride concentrations were unchanged ( - Fig. 4A, - Supplementary Fig. S1D, available in the online version). Cholesterol VLDL, HDL, and LDL fractions were also all reduced under both treatment groups ( - Fig. 4B-D). Based upon the reported effects of Gal-2 in $\mathrm{IBD},{ }^{8}$ we studied the intestines but there were no abnormal- ities, in particular no signs of IBD ( - Supplementary Fig. S1G, S1H).

Next, the level of hepatosteatosis was graded. High levels of steatosis would be expected following a HCD but there were no differences between treatment groups (-Supplementary Fig. S3C-S3F, available in the online version). ABCA-1 and ABCG-1 expression was assessed in the livers of treated mice, and the expression levels were also unchanged (- Supplementary Fig. S3A, S3B, available in the online version). 

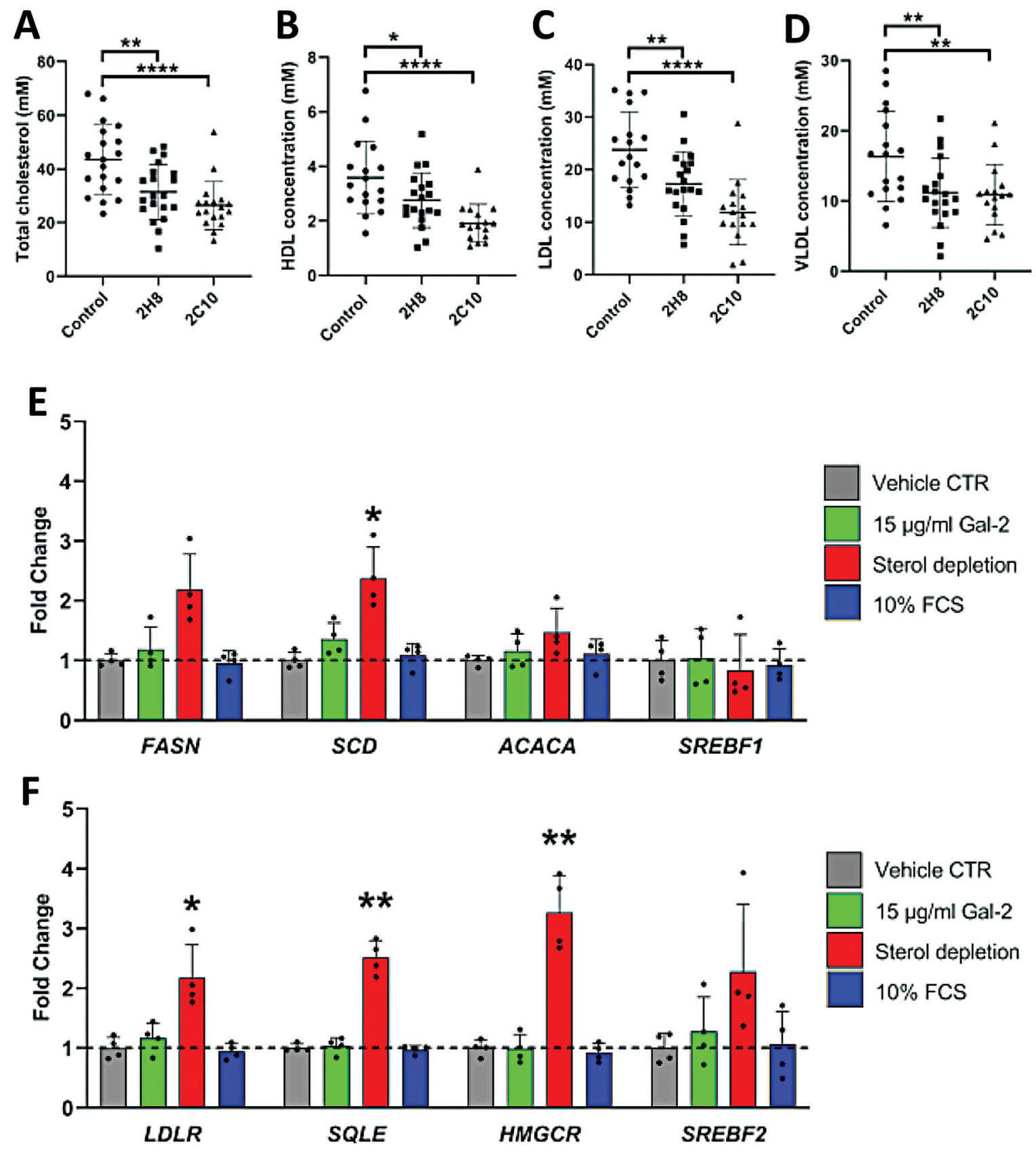

Fig. 4 Serum cholesterol concentrations are reduced in anti-galectin-2 (Gal-2) treated mice while the main cholesterol genesis pathways in the liver are unaltered. Total serum cholesterol concentrations in anti-Gal-2 treated mice are shown (A) under control, $2 \mathrm{H} 8$, and $2 \mathrm{C} 10$ treatments with fractions therein of high-density lipoprotein (HDL) (B), low-density lipoprotein (LDL) (C), and very low-density lipoprotein (VLDL) (D) concentrations also shown. The fold change on four key SREBP1 (E) and SREBP2 (F) targets is shown under various conditions including stimulation with galectin is also shown. Mean values with standard deviation (A-F). $n=17(\mathrm{~A}-\mathrm{D}), n=4(\mathrm{E}, \mathrm{F}) .{ }^{*} p \leq 0.05 .{ }^{* *} p \leq 0.01$. ${ }^{* * *} p \leq 0.001$. ${ }^{* * * *} p \leq 0.0001$.

To investigate the potential effects of Gal-2 on cholesterol metabolism in more detail, hepatocyte-like HepG2 cells were treated with recombinant Gal-2 protein $(15 \mu \mathrm{g} / \mathrm{mL})$ for 24 hours, and the expression of key SREBP-regulated genes in cholesterol metabolism were determined. ${ }^{18}$ Specifically, we quantified the levels of $L D L R, S Q L E, H M G C R$, and SREBF2 (SREBP2 targets) and of FASN, SCD1, ACACA, and SREBF1
(SREBP1 targets). As anticipated, expression of these genes was sensitive to sterol depletion, but was unaltered following Gal-2 treatment ( - Fig. 4E, F).

In summary, these data show that serum cholesterol and cholesterol fractions are strongly reduced by anti-Gal-2 treatment, while SREBP gene expression in hepatocytes, steatosis, and $\mathrm{ABCA} / \mathrm{G}-1$ levels appear unchanged. 


\section{Discussion}

The current study demonstrates that treatment with $2 \mathrm{H} 8$ and 2C10 anti-Gal-2 nanobodies reduces atherosclerosis burden in mice by 25 and 36\%, respectively, despite treatment being initiated during established atherosclerosis. Nanobody treatment slowed the progression of existing atherosclerosis in addition to reducing plaque area. Furthermore, plaques of anti-Gal-2 treated mice contained more VSMCs, and an increase in CD206+ anti-inflammatory macrophages, suggesting that treatment also induces a more favorable anti-inflammatory plaque phenotype.

In a previous study by Hollander et al, ${ }^{13} 2$-week treatment with $2 \mathrm{H} 8$ and 2C10 anti-Gal-2 nanobodies improved arteriogenesis in a hind limb model in $A p o E^{-/-}$mice but failed to reduce atherosclerotic burden. In contrast, we administered anti-Gal-2 treatment for 6 weeks instead of 2 . Since atherosclerosis is a slowly developing disease, the difference in treatment duration may explain this difference.

Our findings reveal the great therapeutic potential of antiGal-2 treatment in ischemic disease, as these nanobodies are able to promote arteriogenesis ${ }^{13}$ while also reducing atherosclerosis. Therefore, these treatments stand in rarefied company as few therapeutics are able to "kill two birds with one stone." For example, monocyte chemoattractant protein-1 (CCL2) improves the arteriogenic response ${ }^{19}$ but also promotes atherosclerosis. ${ }^{19}$ Relatedly, granulocyte-macrophage colony-stimulating factor is proarteriogenic ${ }^{20}$ and also proatherosclerotic. ${ }^{21}$

In the present study, we utilized anti-Gal-2 nanobodies, which are a special single-domain antibody. Camelids and cartilaginous fishes both produce single-domain antibodies, however, owing to their simpler handling, camelids have found favor in research applications. ${ }^{15}$ Specifically for our study we use llama-derived nanobodies which have a single domainthe variable domains of heavy chains, meaning they are more soluble, stable, and easier to produce than conventional antibodies. ${ }^{15}$ Nanobodies lack an Fc-region yet retain functional diversity and can be manipulated to multivalent forms to increase functional affinity and prolong plasma half-life. ${ }^{22}$ Additionally, these nanobodies can potentially be delivered orally which would allow for easier administration as a future therapy. ${ }^{15}$ At the outset, the $2 \mathrm{H} 8$ and $2 \mathrm{C} 10$ clones were selected based upon affinity and specificity for Gal-2. While Hollander et al found the clones were equally effective in arteriogenesis, our data show that 2C10 has much stronger effects on atherosclerosis than $2 \mathrm{H} 8$. The reasons likely lie in the slightly different epitopes which the antibodies target, during their production there is a panning method designed to select the clone with the highest binding affinity and specificity. Hollander et al report that the 2H8 and 2C10 nanobody clones have different recognition domains; however, there are currently no functional data describing exactly what effects these differences may have, and this should form a focus of future studies.

The increase in $\alpha \mathrm{SMA}+$ cells in plaques of $2 \mathrm{C} 10$ treated mice suggests that anti-Gal-2 treatment induces atherosclerotic plaque stability ${ }^{23}$ and supports our qualitative analysis. Our in vitro data show that despite having no effect on proliferation, Gal-2 is able to induce inflammation in VSMCs. In accordance with earlier reports, ${ }^{11-13}$ we demonstrate that anti-Gal-2 treatment can alter macrophages toward a more anti-inflammatory profile. The plaques of 2C10 treated mice showed an increase in the amount of CD206+ macrophages although absolute plaque macrophage content was unchanged. CD206 is a marker for anti-inflammatory "M2" macrophages which are known to reduce atherosclerosis and increase plaque stability. ${ }^{24}$ Other plaque cell types including $\mathrm{T}$ and $\mathrm{B}$ cells were unaffected by treatment. These results show that Gal-2 inhibition via nanobody treatment holds the potential to reduce VSMC inflammation and increase M2 macrophage polarization, resulting in reductions in atherosclerosis progression and more plaque stability.

An unexpected finding of long-term anti-Gal-2 treatment was the reduction of plasma cholesterol levels. Although we performed multiple analyses to decipher underlying mechanisms, we found no explanation for this phenomenon. Mice did not exhibit weight loss and histological analyses revealed no changes in HCD-induced hepatosteatosis or hepatic inflammation. However, the prolonged and high level of cholesterol in the diets of our mice may have "overwhelmed" any phenotype, therefore we cannot exclude that examining livers earlier in the experimental timeline might have revealed differences between treated and untreated animals.

Additionally, the intestines had normal morphology and showed no signs of inflammation that may have otherwise impinged cholesterol uptake (-Supplementary Fig. S2G-S2I, available in the online version). Furthermore, hepatocyte stimulation with Gal-2 also showed no effects on cholesterol synthesis or efflux. However, in the atherosclerotic plaque, macrophages showed a decrease in ABCA-1 and an increase in ABCG-1 following 2C10 treatment. ABCA-1 is a ubiquitously expressed cholesterol efflux protein responsible for reverse cholesterol transport of lipid-poor apolipoproteins specifically (apoA1 and ApoE) and resultant HDL production. ABCG-1 has a very similar role but is thought to be less specific, it can promote efflux of LDL as well as HDL cholesterol. ${ }^{25}$

Expression of both proteins would be expected to increase and not decrease, making our results somewhat contradictory of each other. The effects of a deficiency in either ABCA-1 or ABCG-1 could be obfuscated by compensatory increases in expression of the other. ${ }^{25}$

These changes may be tissue specific, however, earlier studies showed macrophage-specific changes in just ABCA-1 result in no changes in atherogenesis, ${ }^{26}$ while deficiencies in both transporters in macrophages were shown to be proatherogenic. ${ }^{27}$ Importantly, we found neither $A B C$ protein expression level was altered in the liver despite earlier works showing a genetic loss of function of $A B C A-1$ is proatherogenic $^{28}$ and liver-specific expression of ABCA-1 is important for protection from atherosclerosis. ${ }^{26}$

Despite this, the changes we found in these transporters could potentially explain the cholesterol phenotype and further studies should seek to unpick the precise molecular mechanisms via knockdown mouse models for example.

In conclusion, our data show anti-Gal-2 nanobodies have a great potential as a future therapeutic option for 
cardiovascular disease (CVD). In this study, we found the 2C10 clone in particular was highly potent in reducing atherosclerosis. In tandem with its proarteriogenic effects, this makes the 2C10 nanobody a suitable candidate to enter the drug development pipeline. It also makes it a unique treatment option for ischemic CVD, due to its proarteriogenic and anti-atherogenic qualities. However, further mechanistic studies further exploring how anti-Gal-2 treatment reduces plaque inflammation and cholesterol levels should be performed.

\section{What is known about this topic?}

- Galectin-2 has numerous cellular functions in immunity and inflammation.

- Arteriogenesis studies show galectin-2 binds to CD14 on the surface of macrophages, polarizing them to a proinflammatory phenotype.

- Anti-galectin-2 nanobodies are proarteriogenic, but their role in atherosclerosis is unknown.

\section{What does this paper add?}

- Inhibition of galectin-2 via nanobodies causes a reduction of plaque size and plaque phenotype in mice.

- Anti-Gal-2 nanobodies polarize intraplaque macrophages to an M2 anti-inflammatory phenotype.

- This study is the first report of a proarteriogenic and anti-atherogenic compound, to further embolden research seeking to utilize nanobodies for therapeutic treatments.

\section{Funding}

This project has received funding from the European Union's Horizon 2020 research and innovation program under the Marie Skłodowska-Curie Actions, grant agreement No. 812699 (to J.K.). We also acknowledge the support from Amsterdam Cardiovascular Sciences for grant support (to M.J.), as well as the Netherlands Cardiovascular Research Initiative: the Dutch Heart Foundation, Dutch Federation of University Medical Centres, the Netherlands Organization for Health Research, and Development and the Royal Netherlands Academy of Sciences for the GENIUS-II project 'Generating the best evidence-based pharmaceutical targets for atherosclerosis' (CVON2017-22 to E.L.). This study was also supported by the Deutsche Forschungsgemeinschaft (CRC 1123 to E.L., P.v.H.).

\section{Conflict of Interest}

M.V. reports having served as advisor for FMC, Amgen, Medice, Otsuka, Vifor, and Kyowa Kirin. Research support from FMC, Amgen, and Vifor. No other conflicts of interest to report.

\section{Acknowledgment}

The authors wish to thank David Coulson for proofreading the manuscript.

\section{References}

1 Gisterå A, Hansson GK. The immunology of atherosclerosis. Nat Rev Nephrol 2017;13(06):368-380

2 Than NG, Romero R, Goodman M, et al. A primate subfamily of galectins expressed at the maternal-fetal interface that promote immune cell death. Proc Natl Acad Sci U S A 2009;106(24): 9731-9736

3 Vasta GR. Galectins as pattern recognition receptors: structure, function, and evolution. Adv Exp Med Biol 2012;946:21-36

4 Loser K, Sturm A, Voskort M, et al. Galectin-2 suppresses contact allergy by inducing apoptosis in activated CD8+ T cells. J Immunol 2009;182(09):5419-5429

5 Rabinovich GA, Toscano MA. Turning 'sweet' on immunity: galectin-glycan interactions in immune tolerance and inflammation. Nat Rev Immunol 2009;9(05):338-352

$6 \mathrm{Si} \mathrm{Y,} \mathrm{Feng} \mathrm{S,} \mathrm{Gao} \mathrm{J,} \mathrm{et} \mathrm{al.} \mathrm{Human} \mathrm{galectin-2} \mathrm{interacts} \mathrm{with}$ carbohydrates and peptides non-classically: new insight from X-ray crystallography and hemagglutination. Acta Biochim Biophys Sin (Shanghai) 2016;48(10):939-947

7 Sturm A, Lensch M, André S, et al. Human galectin-2: novel inducer of $\mathrm{T}$ cell apoptosis with distinct profile of caspase activation. J Immunol 2004;173(06):3825-3837

8 Paclik D, Berndt U, Guzy C, et al. Galectin-2 induces apoptosis of lamina propria $\mathrm{T}$ lymphocytes and ameliorates acute and chronic experimental colitis in mice. J Mol Med (Berl) 2008;86(12): 1395-1406

9 Meier P, Gloekler S, Zbinden R, et al. Beneficial effect of recruitable collaterals: a 10 -year follow-up study in patients with stable coronary artery disease undergoing quantitative collateral measurements. Circulation 2007;116(09):975-983

10 Hollander MR, Horrevoets AJG, van Royen N. Cellular and pharmacological targets to induce coronary arteriogenesis. Curr Cardiol Rev 2014;10(01):29-37

11 van der Laan AM, Schirmer SH, de Vries MR, et al. Galectin-2 expression is dependent on the rs7291467 polymorphism and acts as an inhibitor of arteriogenesis. Eur Heart J 2012;33(09): 1076-1084

12 Yıldırım C, Vogel DYS, Hollander MR, et al. Galectin-2 induces a proinflammatory, anti-arteriogenic phenotype in monocytes and macrophages. PLoS One 2015;10(04):e0124347

13 Hollander MR, Jansen MF, Hopman LHGA, et al. Stimulation of collateral vessel growth by inhibition of galectin 2 in mice using a single-domain llama-derived antibody. J Am Heart Assoc 2019;8 (20):e012806

14 Ikeda S, Tanaka N, Arai T, Chida K, Muramatsu M, Sawabe M. Polymorphisms of LTA, LGALS2, and PSMA6 genes and coronary atherosclerosis: a pathological study of 1503 consecutive autopsy cases. Atherosclerosis 2012;221(02):458-460

15 Harmsen MM, De Haard HJ. Properties, production, and applications of camelid single-domain antibody fragments. Appl Microbiol Biotechnol 2007;77(01):13-22

16 Virmani R, Kolodgie FD, Burke AP, Farb A, Schwartz SM. Lessons from sudden coronary death: a comprehensive morphological classification scheme for atherosclerotic lesions. Arterioscler Thromb Vasc Biol 2000;20(05):1262-1275

17 Fedoseienko A, Wijers M, Wolters JC, et al. The COMMD family regulates plasma LDL levels and attenuates atherosclerosis through stabilizing the CCC complex in endosomal LDLR trafficking. Circ Res 2018;122(12):1648-1660

18 Goldstein JL, DeBose-Boyd RA, Brown MS. Protein sensors for membrane sterols. Cell 2006;124(01):35-46

19 Lin J, Kakkar V, Lu X. Impact of MCP-1 in atherosclerosis. Curr Pharm Des 2014;20(28):4580-4588

20 Grundmann S, Hoefer I, Ulusans S, et al. Granulocyte-macrophage colony-stimulating factor stimulates arteriogenesis in a pig model of peripheral artery disease using clinically applicable infusion pumps. J Vasc Surg 2006;43(06):1263-1269 
21 Subramanian M, Thorp E, Tabas I. Identification of a non-growth factor role for GM-CSF in advanced atherosclerosis: promotion of macrophage apoptosis and plaque necrosis through IL-23 signaling. Circ Res 2015;116(02):e13-e24

22 Sparkes A, De Baetselier P, Brys L, et al. Novel half-life extended anti-MIF nanobodies protect against endotoxic shock. FASEB J 2018;32(06):3411-3422

23 Harman JL, Jørgensen HF. The role of smooth muscle cells in plaque stability: therapeutic targeting potential. Br J Pharmacol 2019;176(19):3741-3753

24 Willemsen L, de Winther MPJ. Macrophage subsets in atherosclerosis as defined by single-cell technologies. J Pathol 2020;250 (05):705-714
25 Yvan-Charvet L, Wang N, Tall AR. Role of HDL, ABCA1, and ABCG1 transporters in cholesterol efflux and immune responses. Arterioscler Thromb Vasc Biol 2010;30(02):139-143

26 Brunham LR, Singaraja RR, Duong M, et al. Tissue-specific roles of ABCA1 influence susceptibility to atherosclerosis. Arterioscler Thromb Vasc Biol 2009;29(04):548-554

27 Westerterp M, Murphy AJ, Wang M, et al. Deficiency of ATPbinding cassette transporters $\mathrm{A} 1$ and $\mathrm{G} 1$ in macrophages increases inflammation and accelerates atherosclerosis in mice. Circ Res 2013;112(11):1456-1465

28 Bochem AE, van Wijk DF, Holleboom AG, et al. ABCA1 mutation carriers with low high-density lipoprotein cholesterol are characterized by a larger atherosclerotic burden. Eur Heart J 2013;34(04):286-291 